\title{
Paroxysmal spasticity of lower extremities as the initial symptom in two siblings with maple syrup urine disease
}

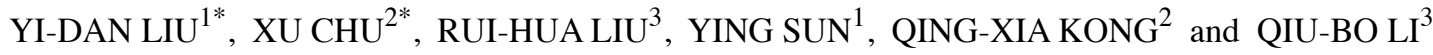 \\ ${ }^{1}$ Cheeloo College of Medicine, Shandong University, Jinan, Shandong 250012; Departments of ${ }^{2}$ Neurology \\ and ${ }^{3}$ Pediatrics, Affiliated Hospital of Jining Medical University, Jining, Shandong 272000, P.R. China
}

Received July 31, 2018; Accepted April 1, 2019

DOI: $10.3892 / \mathrm{mmr} .2019 .10133$

\begin{abstract}
Maple syrup urine disease (MSUD) is a rare autosomal recessive metabolic disorder caused by mutations in genes that encode subunits of the branched-chain $\alpha$-ketoacid dehydrogenase (BCKD) complex. Impairment of the BCKD complex results in an abnormal accumulation of branched-chain amino acids and their corresponding branched-chain keto acids in the blood and cerebrospinal fluid, which are neurovirulent and may become life-threatening. An 11-day-old boy was admitted to the hospital with paroxysmal spasticity of lower extremities. Of note, his 10 -year-old sister presented similar symptoms during the neonatal period, and her condition was diagnosed as MSUD when she was 1.5 years old. Genetic screening was performed, and the boy and his sister exhibited two novel compound heterozygous mutations in the branched chain keto acid dehydrogenase E1 subunit $\beta$ (BCKDHB) gene: A substitution from guanine to adenine in the coding region at position $1,076(\mathrm{c} .1,076 \mathrm{G}>\mathrm{A})$ in exon 10 and a deletion of a thymine at position 705 (c.705delT) in exon 6 . The missense mutation c.1076G $>$ A results in an amino acid substitution from arginine to lysine at position 359 (p.Arg359Lys), whereas the mutation c.705delT results in the replacement of a cysteine at position 235 with a stop codon (p.Cys235Ter). Neither of the BCKDHB alleles in the compound heterozygote patients is able to generate normal E1 $\beta$ subunits, resulting in a possible impairment of the activity of the BCKD complex. In the present study, it was hypothesized that the two novel
\end{abstract}

Correspondence to: Dr Qing-Xia Kong, Department of Neurology, Affiliated Hospital of Jining Medical University, 89 Guhuai Road, Jining, Shandong 272000, P.R. China

E-mail: kxdqy8@sohu.com

Dr Qiu-Bo Li, Department of Pediatrics, Affiliated Hospital of Jining Medical University, 89 Guhuai Road, Jining, Shandong 272000, P.R. China

E-mail: lqb0072@sohu.com

${ }^{*}$ Contributed equally

Key words: maple syrup urine disease, branched chain keto acid dehydrogenase E1 subunit $\beta$ gene, mutation, newborn, encephalopathy heterozygous mutations in the BCKDHB gene found in the Chinese family may be responsible for the phenotype of the two siblings with MSUD.

\section{Introduction}

In the wide array of inherited metabolic disorders, maple syrup urine disease (MSUD; Online Mendelian Inheritance in Man no. 248600; https://www.omim.org/entry/248600) has attracted increasing attention due to the potential neurological damage caused by this disorder (1). MSUD was first reported by Menkes et al (2) in 1954. MSUD is a rare autosomal recessive disorder with an incidence of 1 in 185,000 children worldwide (3). However, the incidence in some regions is expected to be higher due to an increased rate of consanguineous marriages (4).

MSUD is characterized by a deficiency of the branched-chain $\alpha$-ketoacid dehydrogenase (BCKD) complex, affecting the metabolism of branched-chain amino acids (BCAAs), including leucine, isoleucine and valine. Increased circulating levels of BCAAs and their corresponding branched-chain keto acids (BCKAs) in blood and cerebrospinal fluid are neurovirulent and may become life-threatening (5). In addition, increases in BCKAs levels result in a characteristic odor of the urine of patients with MSUD, reminiscent of maple syrup (6).

The BCKD complex consists of three catalytic components: E1, E2 and E3. E1 is a branched-chain $\alpha$-ketoacid decarboxylase, consisting of two E1 $\alpha$ and two E1 $\beta$ subunits, encoded by the branched chain keto acid dehydrogenase E1, $\alpha$ polypeptide (BCKDHA) and by the branched chain keto acid dehydrogenase $\mathrm{E} 1, \beta$ polypeptide (BCKDHB) genes, respectively. E2 is a dihydrolipoyl transacylase encoded by the dihydrolipoamide branched chain transacylase E2 (DBT) gene. E3, a dihydrolipoamide dehydrogenase, is encoded by the dihydrolipoamide dehydrogenase (DLD) gene (7). Mutations in any of the genes that encode components of the BCKD complex may result in MSUD. In the Chinese population, numerous genetic variations have been previously reported, primarily in the BCKDHA and $\mathrm{BCKDHB}$ genes, and certain variations have been reported in the DBT gene (8).

The present study examined the clinical features and genetic variations of two siblings with MSUD in a Chinese family. 


\section{Materials and methods}

Clinical data. The present study examined a Chinese family with five members (Fig. 1). The 10-year-old daughter of the family was admitted to the County Hospital of Juye, Heze in November 2007 with paroxysmal spasticity of lower limbs, when she was 7 days old. Additional clinical manifestations included poor feeding, vomiting and lethargy. Initially, no definitive diagnosis was given, due to the limited medical resources of the local hospital. At the age of 4 months, the girl was misdiagnosed with developmental dysplasia of the hip due to limited leg motor activity, and she was treated with a Pavlik harness for 9 months. However, the clinical symptoms did not improve. At the age of 1.5 years, the girl was diagnosed with MSUD in April 2009 at Peking University First Hospital, Beijing following tandem mass spectrometry (MS/MS) performed in blood samples and gas chromatography combined with mass spectrometry (GC/MS) performed using urine samples. MS/MS analysis in 2009 revealed that the concentrations of leucine and isoleucine, and valine were 635.26 and $530.10 \mu \mathrm{mol} / 1$, respectively. The BCAAs levels in the blood were significantly higher than normal (9). GC/MS analysis identified elevated levels of organic acids in the urine, including 2-OH-isovaleric, 2-keto-isovaleric and acetoacetic acids.

The parents of the patient were healthy and non-consanguineous, without significant disorders (cardiovascular, neurological, endocrine, digestive, respiratory or genitourinary) in their family history. The first son of the family was a year younger than the daughter, and of good health from birth to the present day.

Their third child was born when the eldest daughter was 10 years old. The 11-day-old male was admitted to the Affiliated Hospital of Jining Medical University, Jining in October 2017, due to paroxysmal spasticity of lower extremities, poor feeding, vomiting and lethargy manifested for 3 days, with a urine odor reminiscent of maple syrup. The symptoms were similar to the symptoms of his sister during the neonatal period. Physical examination identified hypermyotonia, poor sucking and rooting reflexes, and incomplete clasping and grasping reflexes. MSUD was a possible diagnosis, and the patient was admitted to the intensive care unit. Convulsions occurred occasionally during hospitalization, characterized by circular movements of the upper limbs and spasticity of lower limbs.

Medical examinations. Metabolic analyses were performed when the youngest son was 11 days old. Peripheral blood (3 drops) was collected from the boy, permeating the filter paper to form dried blood spots, and sent to Shanghai ADICON Clinical Laboratories, Inc. The levels of blood amino acids, including BCAAs (valine and leucine), were measured via MS/MS. Dried blood spot samples with a diameter of $3 \mathrm{~mm}$ were placed in microporous plates. The extraction and derivatization procedures were performed using an Amino Acid and Carnitine Tandem Mass Spectrometry kit (Fenghua Biotech Holding Co., Ltd.) according to the manufacturer's protocol. Samples were analyzed by Shanghai ADICON Clinical Laboratories, Inc. using a TQD LC-MS/MS system (Waters Corporation) and
ChemoView software (SCIEX). The following day, fresh morning urine was collected from the patient, permeating the filter paper; the sample was left to dry naturally and then sent to Shanghai ADICON Clinical Laboratories, Inc. (Shanghai, China) for analysis of the levels of organic acids in urine via gas chromatography-MS, using the GC-MS-QP2010plus analyzer (Shimadzu Corporation, Kyoto, Japan) and the Inborn Errors of Metabolism Screening System software (Shimadzu Corporation). When the boy was 1 month old, these metabolic analyses were repeated.

At the age of 13 days, the boy underwent cerebral magnetic resonance imaging (MRI) and video-electroencephalogram (EEG). For his sister, MRI and video-EEG were also performed when she was 10 years old. The revised Wechsler intelligence scale for children was used to assess intelligence (10).

Genetic screening. To identify the genetic etiology of the disease, genetic screening was performed when the youngest son was 2.5 months old. Peripheral blood samples $(2 \mathrm{ml})$ were collected from each member of the family and were sent to Beijing Kangso Medical Inspection, Co., Ltd. (Beijing, China) for gene panel testing.

The FlexiGene DNA kit (Qiagen GmbH, Hilden, Germany) was used to extract genomic DNA from the blood samples, and the procedures were performed according to the manufacturer's protocol. To construct the DNA library, genomic DNA samples were fragmented into 150-300 bp DNA fragments by an ultrasonic processor (20 cycles of $30 \mathrm{sec}$ on, 30 sec off; Bioruptor ${ }^{\circledR}$ Plus Water Cooler; Diagenode). Adaptors were ligated to both ends, and the cohesive ends were removed. Then, the DNA library was amplified using a PrimeSTAR HS DNA Polymerase (New England BioLabs, Inc., Ipswich, MA, USA) under the following thermocycling conditions: Initial denaturation at $95^{\circ} \mathrm{C}$ for $3 \mathrm{~min}$, followed by 6 cycles of $20 \mathrm{sec}$ at $98^{\circ} \mathrm{C}, 15 \mathrm{sec}$ at $62^{\circ} \mathrm{C}$ and $30 \mathrm{sec}$ at $72^{\circ} \mathrm{C}$, with a final extension at $72^{\circ} \mathrm{C}$ for $5 \mathrm{~min}$ (forward primer, $5^{\prime}$-AATGATACGGCGACCACC GAGATCTACACACACTCTTTCCCTACACGACGCTCT TCCGATC-s-T-3' and reverse primer, 5'-CAAGCAGAAGAC GGCATACGAGAT[i7]GTGACTGGAGTTCAGACGTGT GCTCTTCCGATC-s-T-3'). PCR products were purified using a nucleic acid purification kit (Agencourt AMPure XP; Beckman Coulter, Inc., Brea, CA, USA) according to the manufacturer's protocol.

A customized gene panel for inherited metabolic diseases was designed, which comprised a total of 324 genes. The DNA fragments were hybridized, isolated and then amplified using the SureSelect Target Enrichment System (Herculase II Fusion Enzyme dNTP combo; Agilent Technologies, Inc., Santa Clara, CA, USA) under the following thermocycling conditions: Initial denaturation at $98^{\circ} \mathrm{C}$ for $2 \mathrm{~min}$, followed by 15 cycles of $30 \mathrm{sec}$ at $98^{\circ} \mathrm{C}, 30 \mathrm{sec}$ at $62^{\circ} \mathrm{C}$ and $1 \mathrm{~min}$ at $72^{\circ} \mathrm{C}$, with a final extension at $72^{\circ} \mathrm{C}$ for $10 \mathrm{~min}$ (forward primer, 5'-AATGATACGGCGACCACCGA-3' and reverse primer, 5'-CAAGCAGAAGACGGCATACGA-3'). Then, the products were purified as aforementioned and quantified. Single-read sequencing was performed using the NextSeq500 (Illumina, Inc., San Diego, CA, USA). Raw data were obtained in FASTQ format and were transformed 
Table I. BCKDHB (NM_183050) primer sequences.

\begin{tabular}{lcl}
\hline Genomic coordinates & Nucleotide substitution & \multicolumn{1}{c}{ Primer sequence (5'-3') } \\
\hline chr6:80881070 & c.705delT & F: CAGCCCTTCTTAGCAGCGAGT \\
& & R: CAGCACCTCCTTCACAGTCAAA \\
chr6:81053418 & c. $1,076 \mathrm{G}>\mathrm{A}$ & F: TGGGGCATGGAGGAATTACA \\
& & R: TCATTTTTCGAAGGGCATCA
\end{tabular}

BCKDHB, branched chain keto acid dehydrogenase E1 subunit $\beta$.

into identifiable base sequences using CASAVA software (version 1.8.2; Illumina, Inc.). Sequences were aligned to GRCh37 (as known as hg19) using Burrow-Wheeler Aligner Version 0.7.15-r1140 (11), and single nucleotide and deletion/insertion polymorphisms analyses were performed to obtain mutation information within the targeted regions using GATK Version 3.6 (12).

The candidate mutated regions in BCKDHB gene were selected for further validation. The primers were designed using the online tool PrimerZ (Release 105; ncbi36.genepipe. ncgm.sinica.edu.tw/primerz/beginDesign.do) and were subsequently synthesized (Tianyi Huiyuan Biotech Co., Ltd.). The sequences of the primers used are listed in Table I. The candidate mutation sites were amplified by PCR using a EasyTaq PCR SuperMix (Beijing TransGen Biotech Co., Ltd., Beijing, China), under the following thermocycling conditions: Initial denaturation at $95^{\circ} \mathrm{C}$ for $10 \mathrm{~min}$, followed by 35 cycles of $30 \mathrm{sec}$ at $95^{\circ} \mathrm{C}, 30 \mathrm{sec}$ at $60^{\circ} \mathrm{C}$ and $45 \mathrm{sec}$ at $72^{\circ} \mathrm{C}$, with a final extension at $72^{\circ} \mathrm{C}$ for $5 \mathrm{~min}$. PCR products were subsequently sequenced by Sanger sequencing.

Bioinformatics analysis. To investigate the effects of the detected variants, bioinformatics analyses were performed. RaptorX (http://raptorx.uchicago.edu) was used to predict the tertiary structures of the mutated and wild-type BCKDHB proteins (13). Then, the impact of a certain mutation on the biological function of BCKDHB protein was investigated using SIFT (Version 2; https://sift.bii.a-star.edu.sg/), PolyPhen2 (Version 2; http://genetics.bwh.harvard.edu/pph2/) and MutationTaster (NCBI 37/Ensembl 69; http://www. mutationtaster.org/).

\section{Results}

MS/MS revealed that the 11-day-old boy's blood levels of leucine and valine were $2,027.40$ and $736.86 \mu \mathrm{mol} / 1$, respectively, notably increased compared with usual levels (9). GC/MS also revealed abnormal increases in the urine levels of organic acids, including 2-OH-isovaleric, 3-OH-isovaleric, 2-keto-isovaleric and 2-keto-3-methylvaleric acids (data not shown). The results of the metabolic analyses were consistent with a diagnosis of MSUD (14).

At the age of 13 days, the cranial MRI of the boy demonstrated large areas of eudipleural long T2 signals with limited diffusion in the frontoparietal white matter, basal ganglia-thalamus, brainstem and cerebellum (Fig. 2). For his sister, cranial MRI revealed eudipleural plaque-like long T2 signals in

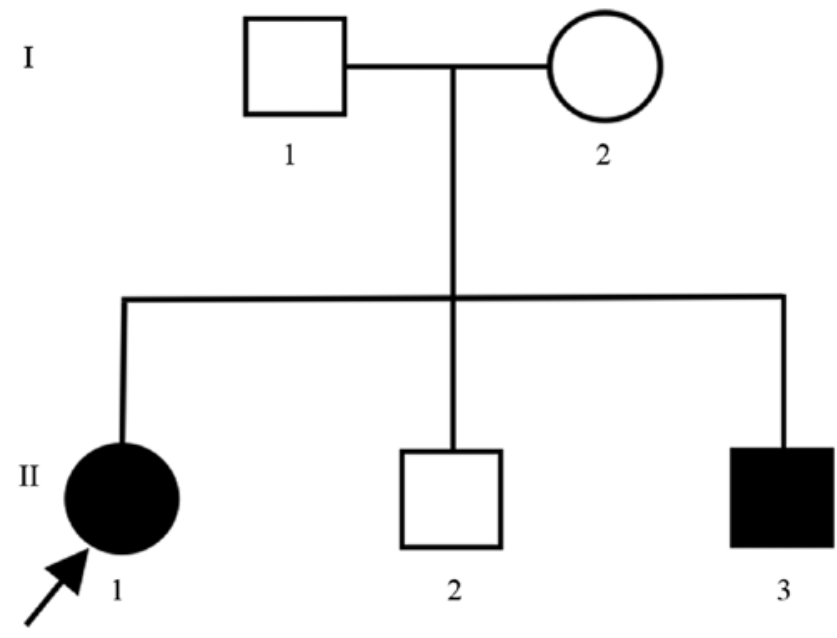

Figure 1. Pedigree chart of the examined family. The Chinese family investigated consisted of five members. I1, the father, 34 years old, healthy. I2, the mother, 34 years old, healthy. II1, the daughter, 10 years old, with MSUD. II2, the first son, 9 years old, healthy. II3, the second son, 11 days old, with MSUD. MSUD, maple syrup urine disease.

bilateral cerebral peduncles, pons, dentate nucleus and medulla (Fig. 3).

Video-EEG was performed when awake and when the 10 -year-old girl was sleeping. During sleep, a small number of spikes and slow wave complexes of medium-high amplitude were identified (Fig. 4A and B, respectively). For the boy, no abnormal waves were identified via video-EEG analysis (data not shown).

Genetic screening identified two compound heterozygous mutations in BCKDHB; a substitution from guanine to adenine in the coding region at position $1,076(\mathrm{c} .1,076 \mathrm{G}>\mathrm{A})$ in exon 10 (Fig. 5) and a deletion of a thymine at position 705 (c.705delT) in exon 6 (Fig. 6). The two siblings had both mutations. Their father, mother and healthy brother had only one of the two heterozygous mutations. The genotypic profiles of the members of the family are presented in Table II.

The mutation c.1,076G $>$ A results in an amino acid substitution from arginine to lysine at position 359 (p.Arg359Lys). SIFT and Polyphen 2 identified that the Arg359Lys mutation may be 'deleterious' and 'damaging', respectively. MutationTaster software indicated that the Arg359Lys mutation could be a 'disease-causing' mutation. The mutation c.705delT results in the replacement of a cysteine at position 235 with a stop codon (p.Cys235Ter). According to the American College of Medical Genetics and Genomics (ACMG) criteria and guidelines (15), 

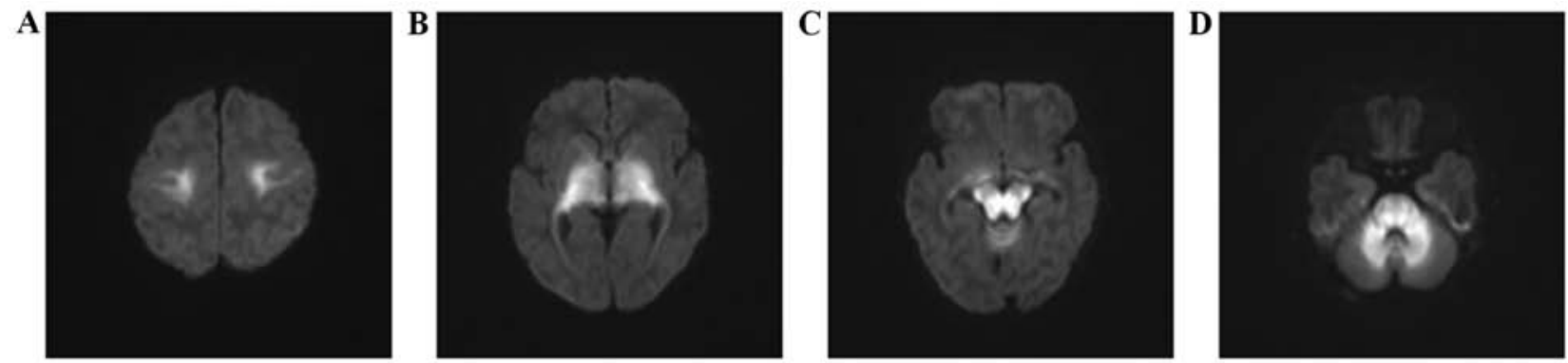

Figure 2. Magnetic resonance diffusion-weighted imaging in the axial plane of the youngest son with MSUD (II3) at 13 days. Large areas of eudipleural long T2 signals with limited diffusion in the (A) frontoparietal white matter, (B) basal ganglia-thalamus, (C) brainstem and (D) cerebellum.
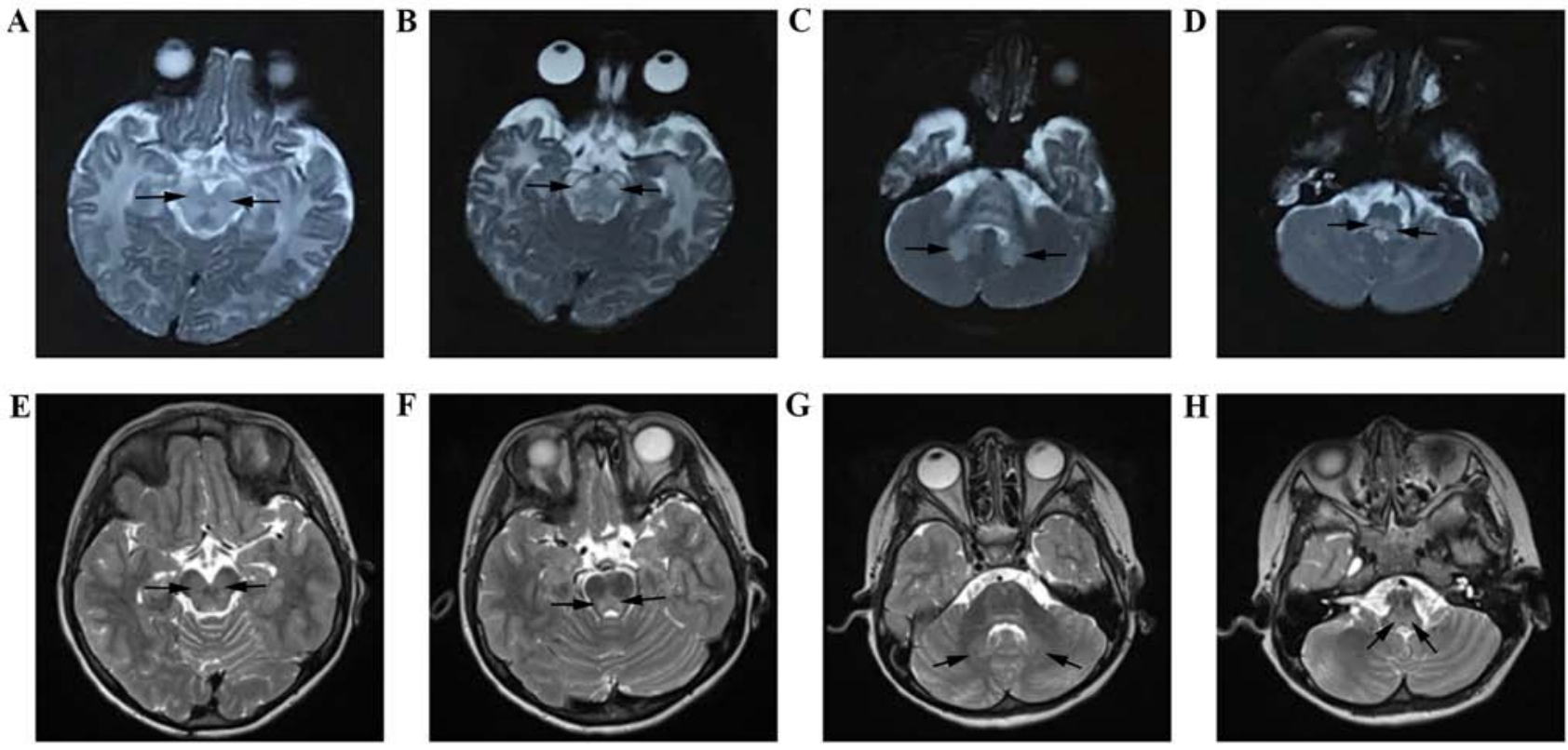

Figure 3. Cranial T2-weighted MRI in the axial plane of the daughter with MSUD (II1). At 7 months of age, the patients exhibited eudipleural plaque-like long T2 signals in (A) bilateral cerebral peduncles, (B) pons, (C) dentate nucleus and (D) medulla (arrows). At 10 years of age, the patients exhibited long T2 signals in (E) bilateral cerebral peduncles, (F) pons, (G) dentate nucleus and (H) medulla (arrows), although certain lesions appeared less severe.

the Cys235Ter mutation is classified as 'likely pathogenic'. To investigate the structural differences between the mutated and wild-type BCKDHB proteins, RaptorX was used to predict the tertiary structures (Fig. 7). Compared with the wild-type BCKDHB protein, there were noticeable differences in the predicted structure of the BCKDHB-mutated protein. Gene mutations were predicted to cause changes in the three-dimensional structure, which could affect the function of the protein.

The 11-day-old boy was promptly treated with a combined therapy. Levocarnitine (500 $\mathrm{mg} /$ day) was used to decrease the circulating levels of malondialdehyde in order to prevent neurological damage. Thiamine (190 $\mathrm{mg}$ three times a day) and BCAA-free medical formula (40 $\mathrm{ml}$ every $3 \mathrm{~h}$ ) were administered to decrease the circulating levels of BCAAs. After the treatment for MSUD, the circulating levels of BCAAs were normal at 1 month of age. At the time of writing, the 6-month-old boy had developed normally without intellectual disability. However, due to a lack of early diagnosis and prompt treatment in the neonatal period, his sister developed an irreversible intellectual disability. Specifically, the 10-year-old girl exhibited the intelligence level of a 3-year-old child and an intelligence quotient $<40$, based on the revised Wechsler intelligence scale for children (10).

\section{Discussion}

In the present study, the two novel heterozygous mutations in the BCKDHB gene found in the family were hypothesized to be responsible for the phenotype of the two children with MSUD. The BCKDHB gene encodes the E1 $\beta$ subunit of the BCKD complex that contains 392 amino acids. There are two Pfam conserved domains in BCKDHB, including a transketolase, pyrimidine binding domain between the amino acid 69 and 247, and a transketolase, C-terminal domain between the amino acid 261 and 382 [(UniProt Knowledgebase; https://www.uniprot.org/ (human BCKDHB)].

The mutation c.1,076G $>$ A results in an amino acid substitution: Lysine replaces arginine at position 359 (p.Arg359Lys). c. $1,076 \mathrm{G}>\mathrm{A}$ is a missense mutation in the $\mathrm{C}$-terminal domain of transketolase, which was identified to be a regulatory domain with a ligand binding site (16). The C-terminal domain has a major role in E1-E2 subunit association, which may be 


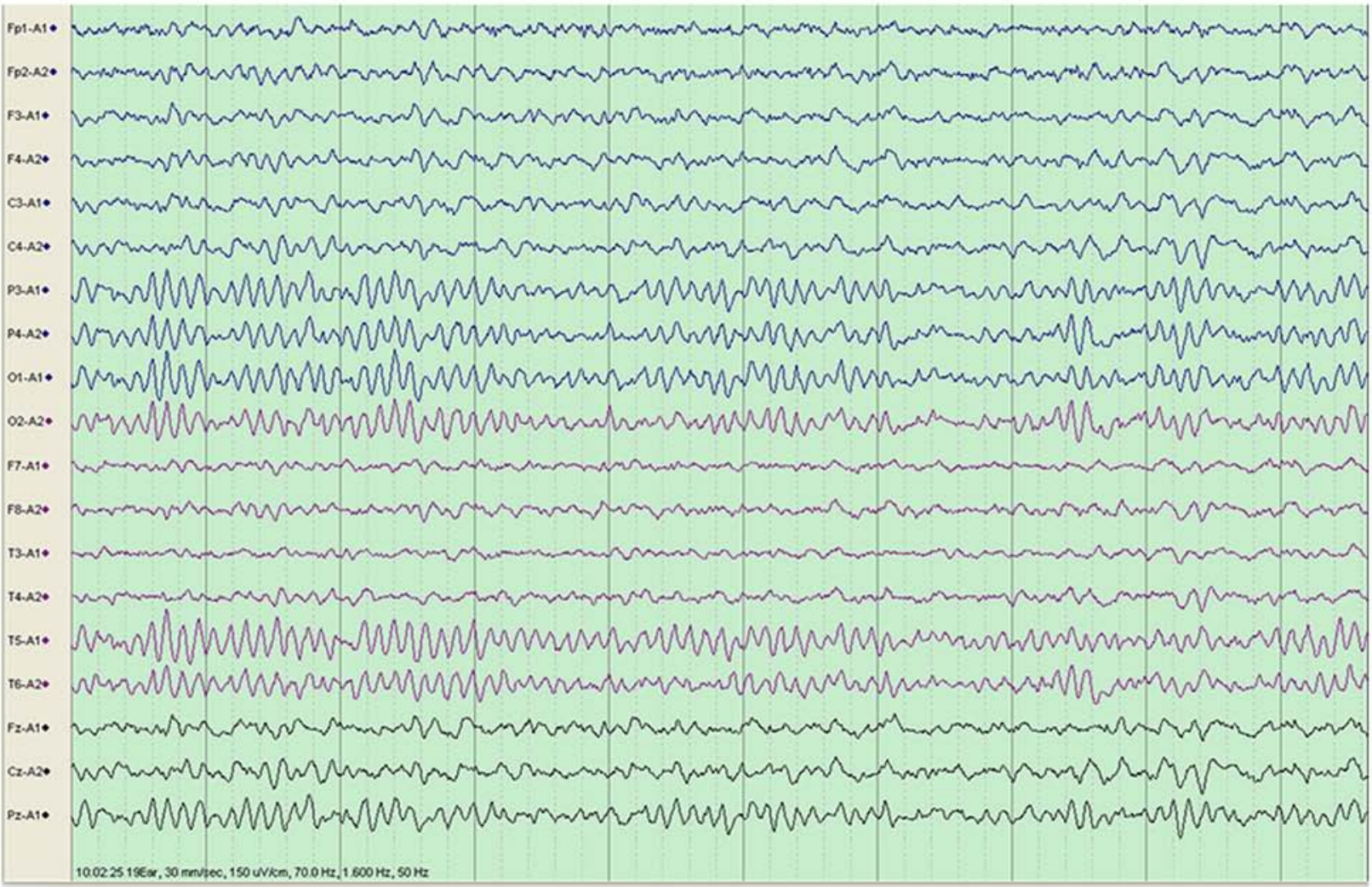

B



Figure 4. Video-EEG of the daughter with MSUD (II1) at the age of 10. (A) Interictal EEG results performed when awake identified a normal $\alpha$ rhythm of 8.5-9.5 Hz, without abnormal waves. (B) EEG results performed when awake identified that all regions exhibited a small number of spikes and slow wave complexes of medium-high amplitude. EEG, electroencephalogram.

destabilized by the mutation in the C-terminal domain (17). In addition, the Arg359 residue is located in the homodimer interface of $\beta-\beta$ ' subunits, which may influence the dimerization of
E1 $\beta$ (18), and mutations in this site may affect the interaction of the E1 $\beta$ subunit with other subunits, altering the assembly of a functional heterotetramer (19). These effects may lead to 
A

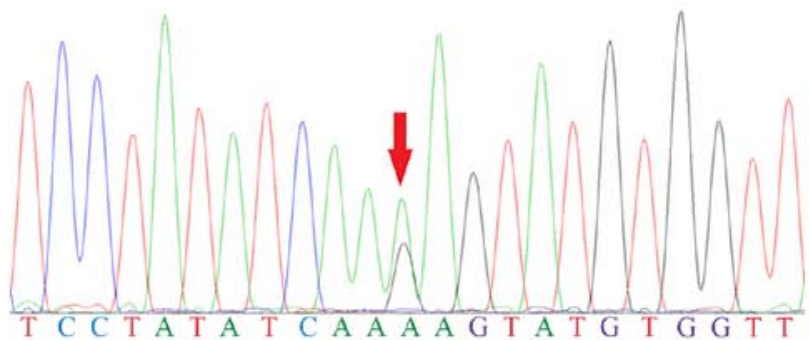

B

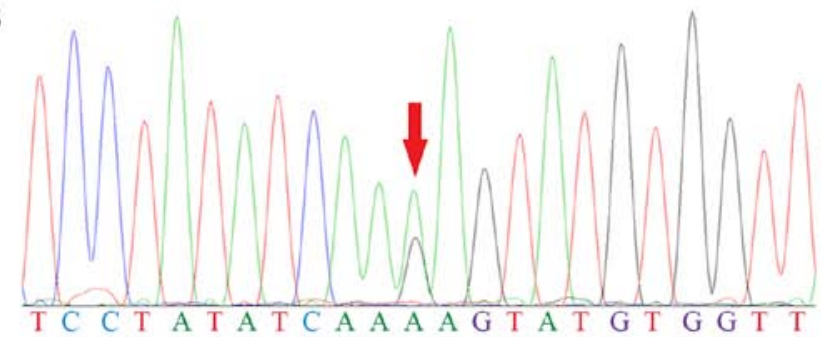

C

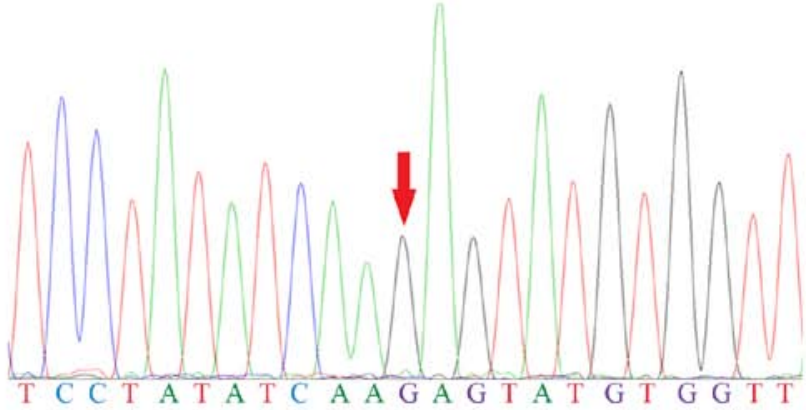

D

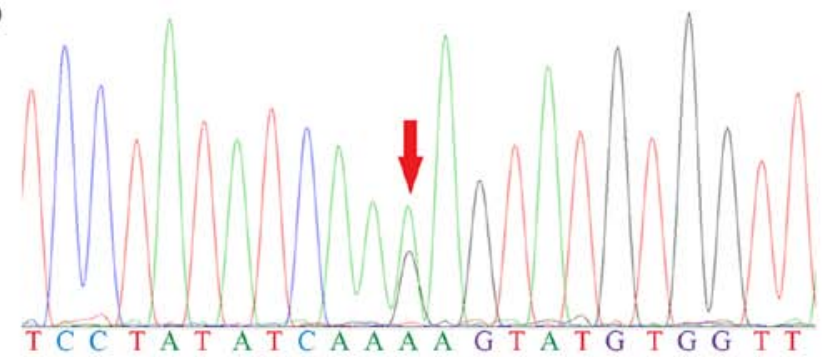

E

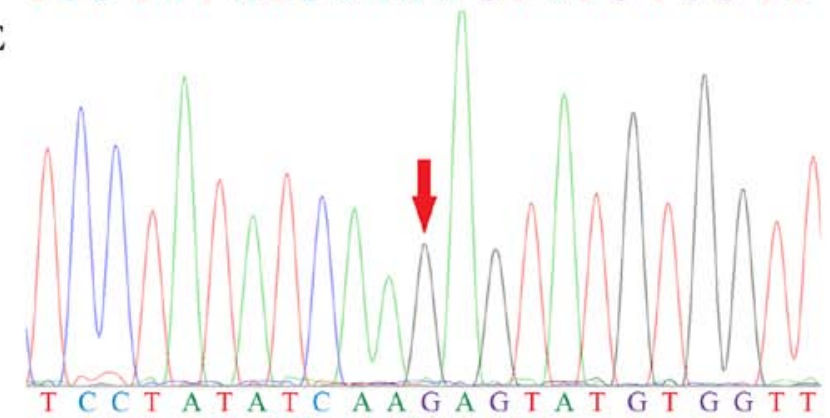

Figure 5. c.1,076G $>$ A mutation identified in exon 10 of the BCKDHB gene. c.1,076G $>$ A mutation in $(\mathrm{A})$ the second son and in $(\mathrm{B})$ the father. (C) Wild-type sequence in the mother. (D) c.1,076G $>$ A mutation in the 10-year-old girl. (E) Wild-type sequence in the healthy son. c.1,076G $>\mathrm{A}$ indicates substitution from guanine to adenine in the coding region at position 1,076. Genomic coordinates, chr6:81053418. BCKDHB, branched chain keto acid dehydrogenase E1 subunit $\beta$.

changes in the structure of the BCKD complex, impairing its BCAAs-degradation activity, thus causing MSUD. SIFT analysis identified that the Arg359Lys mutation may be deleterious. PolyPhen 2 predicted that the mutation was possibly damaging. In addition, MutationTaster indicated that Arg359Lys may be
A

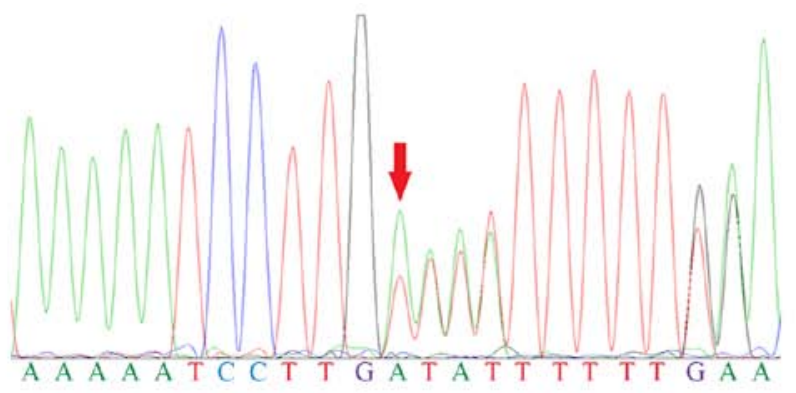

B

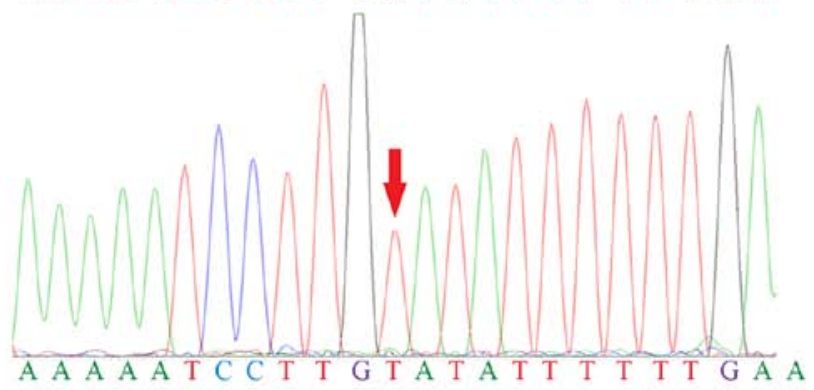

C

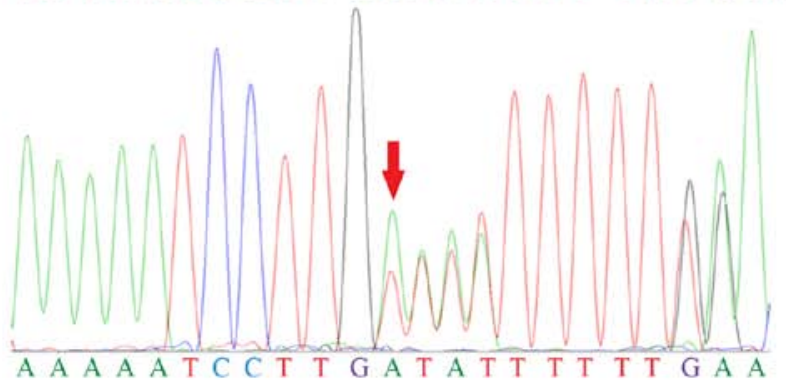

D

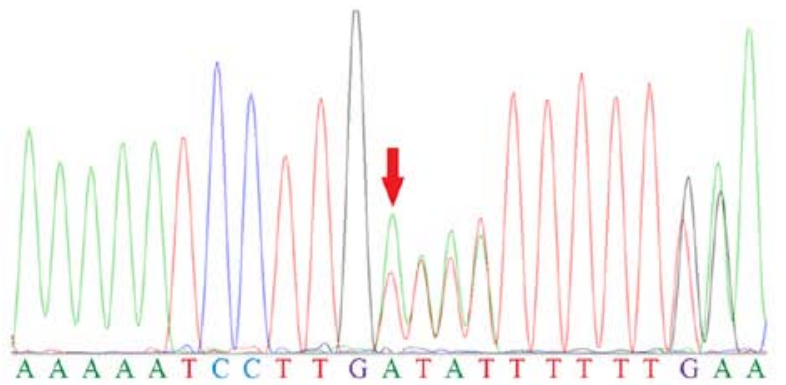

E

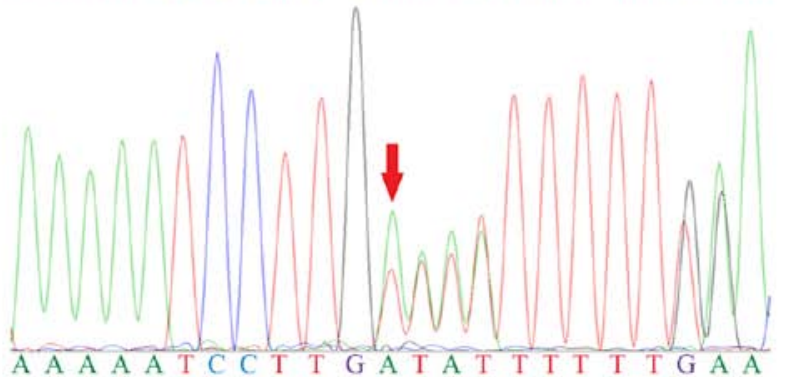

Figure 6. c.705delT mutation identified in exon 6 of the BCKDHB gene. (A) c.705delT mutation in the second son. (B) Wild-type sequence in the father. c.705delT mutation in the (C) mother, in the (D) 10-year-old girl and in the (E) healthy son. c.705delT indicates deletion of thymine at position 705. Genomic coordinates, chr6:80881070. BCKDHB, branched chain keto acid dehydrogenase E1 subunit $\beta$.

a disease-causing mutation. Therefore, Arg359Lys may lead to an unfavorable alteration in the BCKD structure.

The mutation c.705delT results in a replacement of a cysteine to a termination codon at position 235 (p.Cys235Ter). Therefore, the synthesis of the peptide chain is terminated 158 amino acids earlier than the wild-type protein. The 
Table II. Genotype of the five family members.

Family member

\begin{tabular}{|c|c|c|c|c|c|}
\hline Type of mutation in BCKDHB & Second son ${ }^{\mathrm{a}}$ & Fatherb & Mother ${ }^{b}$ & Daughter ${ }^{\mathrm{a}}$ & First son ${ }^{\mathrm{b}}$ \\
\hline c. $1,076 \mathrm{G}>\mathrm{A}$ & Heterozygosis & Heterozygosis & WT & Heterozygosis & WT \\
\hline c.705delT & Heterozygosis & WT & Heterozygosis & Heterozygosis & Heterozygosis \\
\hline
\end{tabular}

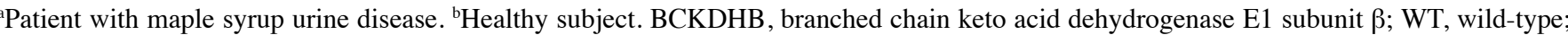
c.705delT, deletion of a thymine at position $705 ;$ c. $1,076 \mathrm{G}>\mathrm{A}$, substitution from guanine to adenine in the coding region at position 1,076 .

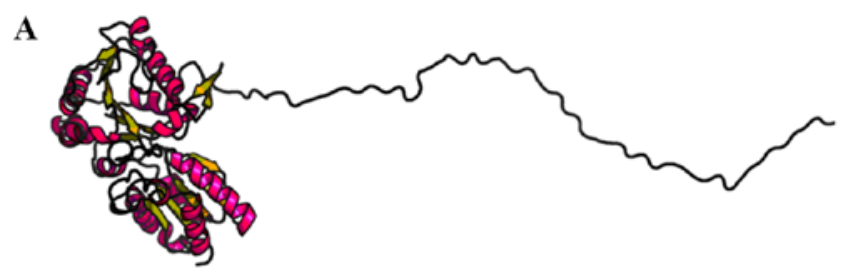

B

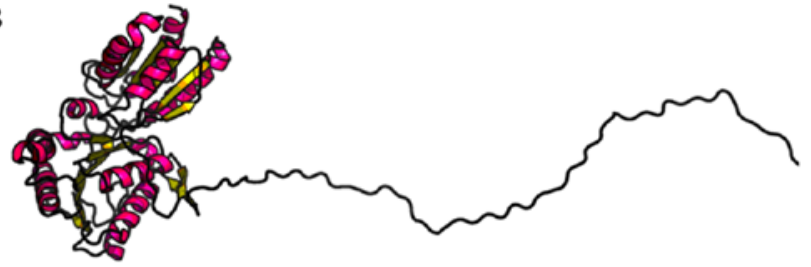

C

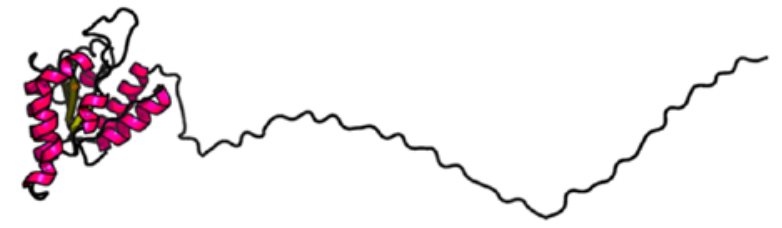

Figure 7. Tertiary structures of the mutated and wild-type BCKDHB proteins predicted by RaptorX. (A) Tertiary structure of the wild-type BCKDHB protein. (B) Predicted tertiary structure of BCKDHB exhibiting an amino acid substitution from arginine to lysine at position 359. (C) Predicted tertiary structure of BCKDHB exhibiting the replacement of a cysteine at position 235 with a stop codon. Differences were identified among the three tertiary structures. BCKDHB, branched chain keto acid dehydrogenase E1 subunit $\beta$.

truncating mutation affects the pyrimidine binding domain, thus inhibiting the transketolase activity (20). Additionally, the protein may lack the $\mathrm{C}$-terminal domain including the $E 1 \beta$ interface segment, thus affecting the E1 $\alpha$-E1 $\beta$ interaction (18). According to the ACMG criteria and guidelines, this variant is classified as 'likely pathogenic'. Collectively, the present results suggested that the c.705delT mutation, which results in the premature termination of the peptide at the amino acid number 235, may influence the function of the BCKD complex.

Neither of the BCKDHB alleles may be able to encode functional E1 $\beta$ subunits in the compound-heterozygote patients, resulting in an impaired activity of BCKD, which may lead to increased circulating levels of BCAAs and BCKAs, thus causing MSUD. Impairment of BCKD function results in accumulation of BCAAs, leucine in particular, which may compete with other essential amino acids for transport through the blood-brain barrier, leading to decreased protein synthesis and demyelination in the brain (21). Furthermore, accumulation of BCKAs may inhibit pyruvate dehydrogenase and $\alpha$-ketogutarate dehydrogenase resulting in Krebs cycle dysfunction, leading to cell swelling and cerebral edema (21). In addition, oxidative stress is involved in neurological damage in MSUD, and leucine may be the principal metabolite causing oxidative brain damage (22).

Although MSUD is an autosomal recessive inherited disorder, compound heterozygous mutations in BCKDHB alleles can induce clinical manifestation of MSUD (23). In the present study, two children with compound heterozygous mutations presented with MSUD, whereas their parents and their brother with only one mutation in one of the two BCKDHB alleles were healthy. In the present study, it was hypothesized that the two mutations, including c.1076G $>$ A and c.705delT, are responsible for the phenotype of the two children with MSUD.

In total, 181 BCKDHB mutations are listed in ClinVar (update in April 2018, ncbi.nlm.nih.gov/clinvar/). Notably, neither of the two mutations identified in the present study have been previously reported in other databases, including the Human Gene Mutation Database (update in January 2018, hgmd.cf.ac.uk/) and PubMed (update in January 2018, ncbi. nlm.nih.gov/pubmed/). MSUD can be classified as type IA, IB, II and III based on mutations in BCKDHA, BCKHB, DBT and DLD, respectively (20). According to the MSUD classification, the two siblings in this Chinese family presented MSUD type IB. The clinical phenotypes of MSUD include classic, intermediate, thiamine-responsive and intermittent forms. Mutations in the genes that encode components of the BCKD complex can result in MSUD. The IA and IB types are associated with the classic form of MSUD, whereas the II and III types are associated with other forms (23).

In addition to the common genetic background, the two siblings had similar clinical manifestations; however, their outcomes were markedly different. The male child had a favorable prognosis because of the early diagnosis and treatment. By contrast, the 10-year-old female exhibited intellectual disability and impaired cognitive functions, due to inadequate treatment during the acute phase of MSUD and poor long-term metabolic management (24).

Early diagnosis of MSUD is important to prevent irreversible brain injury. A maple syrup-like odor in urine and cerumen may indicate MSUD (25). MSUD may be suspected if early signs of metabolic decompensation, including vomiting, poor feeding, diarrhea, fever and lethargy, or neurological features, such as altered level of consciousness, 
headaches, ataxia, dystonia and seizures, are observed (5). In the two siblings, paroxysmal spasticity of lower extremities was the initial symptom. In addition, cerebral MRI results showed restricted diffusion in the myelinated areas on the diffusion-weighted imaging, and this phenotype was identified to be an indicator of MSUD in newborns (26). Additionally, adolescents and young adults with MSUD exhibit increased signals on T2-weighted imaging (27). Notably, the definitive diagnosis of MSUD requires MS/MS and GC/MS analyses, and genetic testing.

Appropriate treatment is crucial in patients with MSUD. Patients with MSUD exhibit defects in the function of the $\mathrm{BCKD}$ complexes that may reduce the interactions between the BCKD complex and its cofactor, thiamine. The decreased affinity for thiamine can be treated by administering high concentrations of thiamine; therefore, thiamine should be used as a therapeutic agent to treat MSUD (28). Another beneficial therapy is L-carnitine supplementation (29). L-carnitine is a potent antioxidant that prevents neurological damage, as molecule is able to cross the blood-brain barrier (30). To maintain normal circulating levels of BCAAs, long-term administration of BCAAs-free medical formula and dietary protein restriction are required (31). Moreover, liver transplantation is an effective treatment, which may reduce the progression of brain damage; however, liver transplantation is not sufficient to reverse pre-existing neurological damages (32). Therefore, early treatment of MSUD is essential to allow normal neuronal development (33).

In summary, the present study investigated clinical and genetic features of two children with MSUD in a Chinese family. In the present study, two novel mutations were identified in BCKDHB, increasing the number of mutations known to be associated with MSUD. Distinct neurocognitive outcomes of the two siblings indicate the importance of early diagnosis and treatment of MSUD.

\section{Acknowledgements}

The authors would like to thank Dr Ying-Ying Su, Dr Sai-Sai Ren, Dr Lin Wang, Dr Xi-Bin Hu and Dr Ting-Ting Bian of the Affiliated Hospital of Jining Medical University for their assistance in collecting the clinical data; Dr Wei Wei of Beijing Kangso Medical Inspection for technical support; and Dr Nan-Nan Jiang of Beijing Children's Hospital, Dr Wei-Yang Li of Jining Medical University and Dr Zhong-Yi Liu of the University of Hong Kong for their assistance in drafting the manuscript.

\section{Funding}

The present study was supported by The Training Program of National Natural Science Foundation of Jining Medical University (grant no. JYP201740) and The Medicine, Health, Science and Technology Development Project of Shandong Province (grant no. 2018WS450).

\section{Availability of data and materials}

The datasets used and/or analyzed during the current study are available from the corresponding author upon reasonable request.

\section{Authors' contributions}

QXK and QBL designed the present study. RHL, YS and YDL collected the clinical data. YDL and XC analyzed the data and wrote the paper. All authors read and approved the final version of the manuscript.

\section{Ethics approval and consent to participate}

The present study was approved by The Ethics Committee of The Affiliated Hospital of Jining Medical University. The parents of the patients provided written informed consent.

\section{Patient consent for publication}

The parents of the patients provided written informed consent for the publication of any associated data and accompanying images.

\section{Competing interests}

The authors declare that they have no competing interests.

\section{References}

1. Sperringer JE, Addington A and Hutson SM: Branched-chain amino acids and brain metabolism. Neurochem Res 42: 1697-1709, 2017.

2. Menkes JH, Hurst PL and Craig JM: A new syndrome: progressive familial infantile cerebral dysfunction associated with an unusual urinary substance. Pediatrics 14: 462-467, 1954.

3. Wasim M, Awan FR, Khan HN, Tawab A, Iqbal M and Ayesha H: Aminoacidopathies: Prevalence, etiology, screening, and treatment options. Biochem Genet 56: 7-21, 2018.

4. Tabbouche O, Saker A and Mountain H: Identification of three novel mutations by studying the molecular genetics of maple syrup urine disease (MSUD) in the lebanese population. Mol Genet Metab Rep 1: 273-279, 2014.

5. Rodan LH, Aldubayan SH, Berry GT and Levy HL: Acute illness protocol for maple syrup urine disease. Pediatr Emerg Care 34: 64-67, 2018.

6. Puckett RL, Lorey F, Rinaldo P, Lipson MH, Matern D, Sowa ME, Levine S, Chang R, Wang RY and Abdenur JE: Maple syrup urine disease: Further evidence that newborn screening may fail to identify variant forms. Mol Genet Metab 100: 136-142, 2010.

7. Su L, Lu Z, Li F, Shao Y, Sheng H, Cai Y and Liu L: Two homozygous mutations in the exon 5 of BCKDHB gene that may cause the classic form of maple syrup urine disease. Metab Brain Dis 32: 765-772, 2017.

8. Li X, Ding Y, Liu Y, Ma Y, Song J, Wang Q, Li M, Qin Y and Yang Y: Eleven novel mutations of the BCKDHA, BCKDHB and DBT genes associated with maple syrup urine disease in the Chinese population: Report on eight cases. Eur J Med Genet 58: 617-623, 2015.

9. Sowell J, Pollard L and Wood T: Quantification of branched-chain amino acids in blood spots and plasma by liquid chromatography tandem mass spectrometry for the diagnosis of maple syrup urine disease. J Sep Sci 34: 631-639, 2011.

10. Wechsler D (ed): Wechsler Intelligence Scale for Children. 5th edition. NCS Pearson, San Antonio, TX, 2014.

11. Li H: Aligning sequence reads, clone sequences and assembly contigs with BWA-MEM. arXiv preprint arXiv: $1303.3997 \mathrm{v} 2$, 2013.

12. McKenna A, Hanna M, Banks E, Sivachenko A, Cibulskis K, Kernytsky A, Garimella K, Altshuler D, Gabriel S, Daly M and DePristo MA: The genome analysis toolkit: A MapReduce framework for analyzing next-generation DNA sequencing data. Genome Res 20: 1297-1303, 2010. 
13. D'Angelo R, Donato L, Venza I, Scimone C, Aragona P and Sidoti A: Possible protective role of the ABCA4 gene c.1268A $>$ G missense variant in Stargardt disease and syndromic retinitis pigmentosa in a Sicilian family: Preliminary data. Int J Mol Med 39: 1011-1020, 2017.

14. Hoffmann GF and Kölker S: Defects in amino acid catabolism and the urea cycle. Handb Clin Neurol 113: 1755-1773, 2013.

15. Richards S, Aziz N, Bale S, Bick D, Das S, Gastier-Foster J, Grody WW, Hegde M, Lyon E, Spector E, et al: Standards and guidelines for the interpretation of sequence variants: A joint consensus recommendation of the American college of medical genetics and genomics and the association for molecular pathology. Genet Med 17: 405-424, 2015.

16. Lindqvist Y, Schneider G, Ermler U and Sundström M: Three-dimensional structure of transketolase, a thiamine diphosphate dependent enzyme, at 2.5 A resolution. EMBO J 11: 2373-2379, 1992.

17. AEvarsson A, Chuang JL, Wynn RM, Turley S, Chuang DT and Hol WG: Crystal structure of human branched-chain alpha-ketoacid dehydrogenase and the molecular basis of multienzyme complex deficiency in maple syrup urine disease. Structure 8: 277-291, 2000

18. Bashyam MD, Chaudhary AK, Sinha M, Nagarajaram HA Devi AR, Bashyam L, Reddy EC and Dalal A: Molecular genetic analysis of MSUD from India reveals mutations causing altered protein truncation affecting the C-termini of E1 $\alpha$ and E1 $\beta$. J Cell Biochem 113: 3122-3132, 2012

19. Edelmann L, Wasserstein MP, Kornreich R, Sansaricq C, Snyderman SE and Diaz GA: Maple syrup urine disease: Identification and carrier-frequency determination of a novel founder mutation in the Ashkenazi Jewish population. Am J Hum Genet 69: 863-868, 2001.

20. Guo Y, Liming L and Jiang L: Two novel compound heterozygous mutations in the BCKDHB gene that cause the intermittent form of maple syrup urine disease. Metab Brain Dis 30: 1395-1400, 2015.

21. Zinnanti WJ, Lazovic J, Griffin K, Skvorak KJ, Paul HS, Homanics GE, Bewley MC, Cheng KC, Lanoue KF and Flanagan JM: Dual mechanism of brain injury and novel treatment strategy in maple syrup urine disease. Brain 132: 903-918, 2009.

22. Sitta A, Ribas GS, Mescka CP, Barschak AG, Wajner M and Vargas CR: Neurological damage in MSUD: The role of oxidative stress. Cell Mol Neurobiol 34: 157-165, 2014.

23. Wang YP, Qi ML, Li TT and Zhao YJ: Two novel mutations in the BCKDHB gene (R170H, Q346R) cause the classic form of maple syrup urine disease (MSUD). Gene 498: 112-115, 2012.
24. Simon E, Schwarz M and Wendel U: Social outcome in adults with maple syrup urine disease (MSUD). J Inherit Metab Dis 30: 264, 2007.

25. Blackburn PR, Gass JM, Vairo FPE, Farnham KM, Atwal HK, Macklin S, Klee EW and Atwal PS: Maple syrup urine disease: Mechanisms and management. Appl Clin Genet 10: 57-66, 2017.

26. Kilicarslan R, Alkan A, Demirkol D, Toprak H and Sharifov R: Maple syrup urine disease: Diffusion-weighted MRI findings during acute metabolic encephalopathic crisis. Jpn J Radiol 30: $522-525,2012$.

27. Schönberger S, Schweiger B, Schwahn B, Schwarz M and Wendel U: Dysmyelination in the brain of adolescents and young adults with maple syrup urine disease. Mol Genet Metab 82: 69-75, 2004.

28. Brown G: Defects of thiamine transport and metabolism. J Inherit Metab Dis 37: 577-585, 2014.

29. Mescka CP, Guerreiro G, Donida B, Marchetti D, Wayhs CA, Ribas GS, Coitinho AS, Wajner M, Dutra-Filho CS and Vargas CR: Investigation of inflammatory profile in MSUD patients: Benefit of L-carnitine supplementation. Metab Brain Dis 30: 1167-1174, 2015.

30. Ribas GS, Vargas CR and Wajner M: L-carnitine supplementation as a potential antioxidant therapy for inherited neurometabolic disorders. Gene 533: 469-476, 2014

31. Li X, Yang Y, Gao Q, Gao M, Lv Y, Dong R, Liu Y, Zhang K and Gai Z: Clinical characteristics and mutation analysis of five Chinese patients with maple syrup urine disease. Metab Brain Dis 33: 741-751, 2018.

32. Díaz VM, Camarena C, de la Vega Á, Martínez-Pardo M, Díaz C, López M, Hernández F, Andrés A and Jara P: Liver transplantation for classical maple syrup urine disease: Long-term follow-up. J Pediatr Gastroenterol Nutr 59: 636-639, 2014.

33. Bouchereau J, Leduc-Leballeur J, Pichard S, Imbard A, Benoist JF, Abi Warde MT, Arnoux JB, Barbier V, Brassier A, Broué $\mathrm{P}$, et al: Neurocognitive profiles in MSUD school-age patients. J Inherit Metab Dis 40: 377-383, 2017.

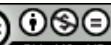

This work is licensed under a Creative Commons Attribution-NonCommercial-NoDerivatives 4.0 International (CC BY-NC-ND 4.0) License. 